\title{
Predictive factors of cardiac rupture in patients with ST-elevation myocardial infarction*
}

\author{
Geng QIAN ${ }^{\dagger 1}$, Chen WU ${ }^{1}$, Yun-dai CHEN ${ }^{\dagger 1}$, Chen-chen $\mathrm{TU}^{2}$, Jin-wen $\mathrm{WANG}^{2}$, Yong-an QIAN ${ }^{3}$ \\ $\left({ }^{1}\right.$ Department of Cardiology, Chinese PLA General Hospital, Beijing 100853, China) \\ ( ${ }^{2}$ Department of Cardiology, Beijing Anzhen Hospital, Capital Medical University, Beijing 100029, China) \\ ( ${ }^{3}$ Department of Cardiology, Lujiang People's Hospital, Anhui 231500, China) \\ 'E-mail: qiangeng9396@263.net; cyundai@126.com
}

Received Apr. 3, 2014; Revision accepted Aug. 7, 2014; Crosschecked Nov. 14, 2014

\begin{abstract}
Cardiac rupture (CR) is a potentially fatal mechanical complication of ST-elevation myocardial infarction (STEMI). We aimed to determine the incidence and risk factors of CR in Chinese STEMI patients. A total of 9798 consecutive STEMI patients from four centers in China were retrospectively analyzed, among which 178 patients had CR. STEMI patients without CR were chosen as a control group. Clinical characteristics were compared between STEMI patients with CR and those without CR. The incidence of CR in STEMI patients was 1.82\%, and the 30-d mortality was up to $61.2 \%$. CR patients were significantly older, more female, and associated with a longer time from onset of pain to hospital admission than their non-CR counterparts $(P<0.001)$. More patients with anterior myocardial infarction $(82.1 \%)$ were found in the CR group, and CR patients had significantly higher heart rates than the control group $((91 \pm 19) \mathrm{bpm}$ vs. $(71 \pm 16) \mathrm{bpm} ; P<0.001)$. In multiple-adjusted models, the independent risk factors of $C R$ were advanced age, female gender, anaemia, increased heart rate, anterior myocardial infarction, increased white blood cell (WBC) count, delayed admission, and renal dysfunction. The level of hemoglobin remained a significant determinant factor of $\mathrm{CR}(\mathrm{OR}(95 \% \mathrm{Cl})$ : $0.82(0.75-0.89) ; P<0.001)$ after adjusting for various potential confounding factors. Counts of WBC also remained a significant determinant of the CR (OR (95\% Cl): $1.08(1.04-1.12) ; P<0.001)$. A number of variables were independently related to $C R$. This study indicated, for the first time, that both hemoglobin and WBC levels were independently correlated with occurrence of CR.
\end{abstract}

Key words: ST-elevation myocardial infarction, Risk factor, Anaemia, Cardiac rupture doi: $10.1631 /$ jzus.B1400095

Document code: A

CLC number: R541.4

\section{Introduction}

Cardiac rupture (CR) after ST-elevation myocardial infarction (STEMI) includes free-wall rupture (FWR), ventricular septal rupture (VSR), and papillary muscle rupture (PMR). Along with the development of primary percutaneous coronary intervention (PCI) and drug therapy, the incidence of $\mathrm{CR}$ has

\footnotetext{
${ }^{\ddagger}$ Corresponding author

* Project supported by the China Capital Clinical Research (No. Z121107001012002)

(i) ORCID: Geng QIAN, http://orcid.org/0000-0002-3238-5860; Yun-dai CHEN, http://orcid.org/0000-0002-3527-5368

(c) Zhejiang University and Springer-Verlag Berlin Heidelberg 2014
}

gradually declined (Figueras et al., 2008). However, there are still a number of deaths caused by CR that could not been determined until autopsy. CR is still one of the common causes of in-hospital deaths for STEMI patients (Markowicz-Pawlus et al., 2007; Figueras et al., 2008). The determination of CR risk factors plays an important role in the prevention of CR. Independent risk factors reported include female gender, advanced age, lower blood pressure, higher heart rate, delayed admission, and thrombolysis (Yip et al., 2003; Okino et al., 2005; French et al., 2010; Lopez-Sendon et al., 2010; Qian et al., 2011). Patients with CR have new clinical characteristics in the era of reperfusion. Up to now there has been a lack of systemic 
research on the assessment of $\mathrm{CR}$ risk factors in Chinese STEMI patients from multiple centers. The objective of our study was to determine the incidence and risk factors of CR in Chinese STEMI patients.

\section{Subjects and methods}

\subsection{Study population}

We retrospectively analyzed a total of 9798 consecutive STEMI patients who were admitted from January 2011 to January 2013 from four centers (Chinese PLA General Hospital, Beijing Anzhen Hospital, Beijing Fuwai Hospital, and Beijing Chao-Yang Hospital). One hundred and seventy-eight of these patients with CR served as a case group, and the others without $\mathrm{CR}$ were chosen as a control group. Diagnosis of STEMI was based on the concurrence of symptoms (chest pain or symptoms compatible with acute heart failure or unexplained syncope) and electrocardiogram findings (ST-segment elevation $\geq 1 \mathrm{~mm}$ in $\geq 2$ inferior leads or $\geq 2 \mathrm{~mm}$ in $\geq 2$ precordial leads). The diagnosis of FWR was based on strict necropsy or electrical mechanical dissociation or pericardial tamponade accompanied with laboratory results (echocardiography showed massive pericardial effusion and diagnostic pericardiocentesis showed bloody fluid). VSR and PMR were established by the emergence of new systolic murmurs and the result of echocardiography. STEMI patients received aspirin, clopidogrel, statin, low molecular weight heparin (LMWH), $\beta$-blockers, and angiotensin converting enzyme inhibitors (ACEIs) or angiotensin receptor blockers (ARBs) according to the STEMI guideline (Kushner et al., 2009), unless there were contraindications to these drugs. We provided a comparative analysis between $\mathrm{CR}$ patients and non-CR patients by clinical manifestations, laboratory profiles, and the treatments during their stay in hospital. Peripheral blood samples were collected from patients within $12 \mathrm{~h}$ of admission for blood routines and biochemistry examinations.

\subsection{Statistical analysis}

Variables following a normal distribution were expressed as mean \pm standard deviation (SD). Variables following a non-normal distribution were expressed as median (interquartile range). The means of normally distributed variables were compared by using Student's $t$-test. For comparisons of the distributions of variables between groups, $\chi^{2}$ analysis was used. The Mann-Whitney $U$ test was used for those with non-normal distribution. Independent CR risk factors were calculated using multivariable logistic regression models (stepwise regression hazards analysis). Statistical analyses were performed using SPSS software (Version 19.0, SPSS Inc., USA). Statistical significance was set at $P<0.05$.

\section{Results}

\subsection{Incidence of CR}

In the 9798 consecutive patients with STEMI, 178 patients (1.82\%) developed CR. Of these CR patients, FWR occurred in 118 patients $(1.20 \%)$, VSR occurred in 49 patients $(0.50 \%)$, and PMR occurred in 11 patients $(0.11 \%)$. The incidence of $\mathrm{CR}$ varied across the four hospital clusters: $2.20 \%(54 / 2450)$ for Chinese PLA General Hospital vs. 1.68\% (43/2560) for Beijing Anzhen Hospital vs. 1.90\% (60/3150) for Beijing Fuwai Hospital vs. 1.44\% (21/1460) for Beijing Chao-Yang Hospital. Despite the improvements in CR treatments such as repair operation and perventricular device closure, the 30-d mortality of CR was still up to $61.2 \%(109 / 178)$ compared with $5.9 \%$ $(567 / 9620)$ in those without CR $(P<0.001)$. And CR accounted for $16.1 \%(109 / 676)$ of all cause in-hospital deaths post-STEMI. In-hospital mortality was higher in patients with FWR (92/118, 78.0\%) than in those with VSR or PMR $(17 / 60,28.3 \%)$. The presence of cardiac tamponade combined with hemodynamic instability was the common finding of FWR $(68 / 118,57.6 \%)$, while sudden unexpected cardiogenic shock or heart failure was the common finding of VSR or PMR (49/60, 81.7\%).

\subsection{Clinical characteristics and treatments before CR}

Demographics, medical histories, clinical features, and treatments in patients with and without CR are shown in Table 1. The female proportion and advanced age proportion in the CR group were significantly higher than those in the control group $(P<0.001)$. The occurrence of hypertension was more frequent in $\mathrm{CR}$ patients $(P<0.001)$, whereas smoking 
occurred less frequently in $\mathrm{CR}$ patients $(P=0.037)$. More patients with anterior myocardial infarction (82.1\%) were found in the CR group. There were also significant trends toward higher heart rate and lower blood pressure in the $\mathrm{CR}$ group $(P<0.001)$. The initial hemoglobin levels of the $\mathrm{CR}$ group were significantly lower than those of the control group $((12.1 \pm 1.8) \mathrm{g} / \mathrm{dl}$ vs. $(13.4 \pm 2.0) \mathrm{g} / \mathrm{dl} ; P<0.001)$, while the white blood cell (WBC) counts were significantly higher in the CR group $\left((11.5 \pm 4.2) \times 10^{9} \mathrm{~L}^{-1}\right.$ vs. $(9.6 \pm 3.7) \times 10^{9} \mathrm{~L}^{-1}$; $P<0.001)$. The $\mathrm{CR}$ patients tended to have longer time from onset of pain to first medical contact (12 (6-22) h vs. $10(4-16) \mathrm{h} ; P<0.001)$. Both left ventricular ejection fraction and Killip class indicated that the CR group had worse heart function. Recommended medications within the first $24 \mathrm{~h}$ including ACEI/ARB and $\beta$-blockers were used less frequently in $\mathrm{CR}$ patients $(P<0.001)$. Furthermore, primary PCI treatment was used more frequently in the non-CR group $(P<0.001)$.

\subsection{Risk factors for post-infarct CR}

Multivariable logistic regression analysis included clinical characteristics on admission (age, gender, heart rate, smoking habit, blood pressure, myocardial infarction location, Killip class, hemoglobin level, count of WBC, time to admission, and eGFR; 9798 patients with complete data). The independent predictors of $\mathrm{CR}$ were as below: female gender (odds ratio (OR) 2.63, 95\% confidence

Table 1 Clinical characteristics and treatments in STEMI patients with and without cardiac rupture (CR)

\begin{tabular}{|c|c|c|c|}
\hline Clinical characteristics and treatment & CR group $(n=178)$ & Control group $(n=9620)$ & $P$ \\
\hline \multicolumn{4}{|l|}{ Demographic characteristics } \\
\hline Male & $98(55.1 \%)$ & $7763(80.7 \%)$ & $<0.001$ \\
\hline Age (year) & $67 \pm 10$ & $61 \pm 13$ & $<0.001$ \\
\hline BMI $\left(\mathrm{kg} / \mathrm{m}^{2}\right)$ & $24.2 \pm 3.3$ & $25.0 \pm 4.0$ & 0.252 \\
\hline Hypertension & $108(60.7 \%)$ & $5069(52.7 \%)$ & 0.037 \\
\hline Diabetes mellitus & $48(27.0 \%)$ & $2309(24.0 \%)$ & 0.866 \\
\hline Hypercholesterolemia & $40(22.5 \%)$ & $1673(17.4 \%)$ & 0.079 \\
\hline Current smoking & $59(33.1 \%)$ & $5099(53.0 \%)$ & $<0.001$ \\
\hline \multicolumn{4}{|l|}{ Clinical presentation in the first $24 \mathrm{~h}$} \\
\hline Anterior myocardial infarction & $146(82.1 \%)$ & $5262(54.7 \%)$ & $<0.001$ \\
\hline $\mathrm{SBP}(\mathrm{mmHg})$ & $111 \pm 21$ & $122 \pm 23$ & $<0.001$ \\
\hline DBP $(\mathrm{mmHg})$ & $70 \pm 13$ & $73 \pm 14$ & 0.014 \\
\hline Pulse (bpm) & $91 \pm 19$ & $77 \pm 16$ & $<0.001$ \\
\hline Killip class III or IV & $75(42.1 \%)$ & $2097(21.8 \%)$ & $<0.001$ \\
\hline Initial white blood cell $\left(\times 10^{9} \mathrm{~L}^{-1}\right)$ & $11.5 \pm 4.2$ & $9.6 \pm 3.7$ & $<0.001$ \\
\hline Initial hemoglobin $(\mathrm{g} / \mathrm{dl})$ & $12.1 \pm 1.8$ & $13.4 \pm 2.0$ & $<0.001$ \\
\hline Time from onset to admission (h) & $12(6-22)$ & $10(4-16)$ & $<0.001$ \\
\hline Initial random glucose $(\mathrm{mmol} / \mathrm{L})$ & $9.9 \pm 5.1$ & $9.2 \pm 3.6$ & 0.131 \\
\hline Initial LDL-C (mmol/L) & $2.73 \pm 0.83$ & $2.81 \pm 1.07$ & 0.424 \\
\hline $\mathrm{eGFR}(\mathrm{ml} / \mathrm{min})$ & $61.6 \pm 24.8$ & $79.6 \pm 23.5$ & $<0.001$ \\
\hline Left ventricular ejection fraction (\%) & $47 \pm 11$ & $52 \pm 11$ & $<0.001$ \\
\hline Left ventricular end diastolic diameter $(\mathrm{mm})$ & $53 \pm 9$ & $49 \pm 6$ & $<0.001$ \\
\hline In-hospital mortality & $109(61.2 \%)$ & $567(5.9 \%)$ & $<0.001$ \\
\hline \multicolumn{4}{|l|}{ Treatments in the first $24 \mathrm{~h}$} \\
\hline Aspirin+thienopyridine & $162(91.0 \%)$ & $9004(93.6 \%)$ & 0.173 \\
\hline$\beta$-Blocker & $121(68.0 \%)$ & $7436(77.3 \%)$ & $<0.001$ \\
\hline ACEI/ARB & $74(41.4 \%)$ & $4598(47.8 \%)$ & 0.009 \\
\hline Statin & $164(92.1 \%)$ & $9120(94.8 \%)$ & 0.119 \\
\hline Primary percutaneous intervention & $54(30.3 \%)$ & $6705(69.7 \%)$ & $<0.001$ \\
\hline Thrombolysis & $31(17.4 \%)$ & $1279(13.3 \%)$ & 0.116 \\
\hline
\end{tabular}

Data are expressed as number of patients (percentage), median (range), or mean \pm SD. BMI: body mass index; SBP: systolic blood pressure; DBP: diastolic blood pressure; LDL-C: low density lipoprotein cholesterol; eGFR: estimated glomerular filtration rate; ACEI/ARB: angiotensin converting enzyme inhibitor or angiotensin receptor blocker 
interval (CI) 1.77-3.92), age per 10-year increase (OR 1.30, 95\% CI 1.10-1.53), heart rate per 10-beat per minute (bpm) increase (OR 1.42, 95\% CI $1.29-1.56$ ), systolic blood pressure (SBP) per 10 -mmHg decrease (OR 1.27, 95\% CI 1.18-1.40), anterior myocardial infarction (OR $3.23,95 \% \mathrm{CI}$ 2.07-5.04), hemoglobin level $\geq 12 \mathrm{~g} / \mathrm{dl}$ (OR $0.49,95 \%$ CI $0.32-0.73$ ), count of WBC (OR $1.09,95 \%$ CI $1.05-1.14$ ), time to admission (OR 1.01, 95\% CI $1.00-1.12$ ), and eGFR $\leq 60 \mathrm{ml} / \mathrm{min}$ (OR $1.98,95 \% \mathrm{CI}$ 1.32-2.97). These are shown in Table 2.

\subsection{New CR predictors: the relationships between results of blood routine and $C R$}

We have explored new risk factors of CR: one was lower hemoglobin level, and the other was higher WBC count. Anemia had a close relationship with gender, age, and renal function, so we analyzed the association between hemoglobin level and occurrence of CR after adjustments for the above confounding factors. We found that the lower level of hemoglobin remained a significant determinant of CR (OR $(95 \% \mathrm{CI})$ : $0.82(0.75-0.89) ; P<0.001 ; c$-statistic value $=0.680)$ after adjusting for various confounding factors. There was no significant interaction between male and female $(P=0.420)$, as shown in Table 3. Higher WBC counts also remained a significant determinant of $\mathrm{CR}$ (OR (95\% CI): 1.08 (1.04-1.12); $P<0.001 ; c$-statistic value $=0.629$ ), as shown in Table 4 . The lower level of hemoglobin was also independently correlated with pericardial effusion $(P<0.001)$. Incidence of $\mathrm{CR}$ increased with the decline of hemoglobin level, while incidence of CR increased with the increase of WBC counts, which is shown in Fig. 1.

Table 2 Univariate and multivariate analyses of clinical characteristics for cardiac rupture (CR) patients

\begin{tabular}{lcccccccc}
\hline \multirow{2}{*}{ Variables } & \multicolumn{3}{c}{ Univariate analysis } & & \multicolumn{3}{c}{ Multivariate analysis } \\
\cline { 2 - 3 } \cline { 7 - 9 } & OR & $95 \%$ CI & $P$ value & & OR & $95 \%$ CI & $P$ value \\
\hline Age per extra 10 years & 1.44 & $1.26-1.63$ & $<0.001$ & & 1.30 & $1.10-1.53$ & $<0.001$ \\
Female gender & 3.42 & $2.52-4.63$ & $<0.001$ & & 2.63 & $1.77-3.92$ & $<0.001$ \\
Current smoking & 0.44 & $0.32-0.60$ & $<0.001$ & & 0.76 & $0.52-1.10$ & 0.143 \\
HR per extra 10 bpm & 1.53 & $1.42-1.65$ & $<0.001$ & & 1.42 & $1.29-1.56$ & $<0.001$ \\
SBP per extra 10 mmHg & 0.79 & $0.74-0.85$ & $<0.001$ & & 0.78 & $0.71-0.85$ & $<0.001$ \\
Anterior myocardial infarction & 3.80 & $2.56-5.63$ & $<0.001$ & & 3.23 & $2.07-5.04$ & $<0.001$ \\
Killip class III or IV & 2.62 & $1.93-3.55$ & $<0.001$ & & 0.85 & $0.57-1.25$ & 0.398 \\
Hemoglobin level $\geq 12 \mathrm{~g} / \mathrm{dl}$ & 0.36 & $0.26-0.49$ & $<0.001$ & & 0.49 & $0.32-0.73$ & $<0.001$ \\
Count of WBC & 1.11 & $1.08-1.15$ & $<0.001$ & & 1.09 & $1.05-1.14$ & $<0.001$ \\
Time to admission & 1.65 & $1.16-2.35$ & & 0.005 & & 1.01 & $1.00-1.02$ & 0.015 \\
eGFR $\leq 60 \mathrm{ml} /$ min & 0.26 & $0.19-0.35$ & $<0.001$ & & 1.98 & $1.32-2.97$ & $<0.001$ \\
\hline
\end{tabular}

HR: heart rate; SBP: systolic blood pressure; WBC: white blood cell; eGFR: estimated glomerular filtration rate

Table 3 Association between anemia and cardiac rupture (CR) evaluated with logistic regression analysis, with adjustments for age, gender, and other confounding factors

\begin{tabular}{|c|c|c|c|c|c|c|}
\hline \multirow{2}{*}{ Gender } & \multirow{2}{*}{$\operatorname{HGB}(\mathrm{g} / \mathrm{dl})$} & \multicolumn{2}{|l|}{ Model 1} & \multicolumn{2}{|l|}{ Model 2} & \multirow{2}{*}{$\begin{array}{l}P \text { value of } \\
\text { interaction }\end{array}$} \\
\hline & & OR $(95 \% \mathrm{CI})$ & $P$ value & OR $(95 \% \mathrm{CI})$ & $P$ value & \\
\hline Male & $13.5 \pm 1.9$ & $0.785(0.717-0.859)$ & $<0.001$ & $0.756(0.655-0.872)$ & $<0.001$ & \multirow{2}{*}{0.420} \\
\hline Female & $12.2 \pm 1.9$ & $0.801(0.726-0.884)$ & $<0.001$ & $0.862(0.739-0.998)$ & 0.045 & \\
\hline
\end{tabular}

Model 1 regression was done with adjustment for age; Model 2 regression was done with adjustment for age, systolic blood pressure, heart rate, estimated glomerular filtration rate, anterior myocardial infarction, and count of white blood cell. HGB: hemoglobin

Table 4 Association between higher count of WBC and cardiac rupture (CR) evaluated with logistic regression analysis, with adjustments for age, gender and other confounding factors

\begin{tabular}{|c|c|c|c|c|c|c|}
\hline \multirow{2}{*}{ Gender } & \multirow{2}{*}{$\begin{array}{c}\text { WBC } \\
\left(\times 10^{9} \mathrm{~L}^{-1}\right)\end{array}$} & \multicolumn{2}{|l|}{ Model 1} & \multicolumn{2}{|l|}{ Model 2} & \multirow{2}{*}{$\begin{array}{l}P \text { value of } \\
\text { interaction }\end{array}$} \\
\hline & & OR $(95 \% \mathrm{CI})$ & $P$ value & OR $(95 \% \mathrm{CI})$ & $P$ value & \\
\hline Male & $9.65 \pm 3.66$ & $1.115(1.063-1.169)$ & $<0.001$ & $1.066(1.012-1.122)$ & $<0.001$ & \multirow{2}{*}{0.480} \\
\hline Female & $9.70 \pm 4.12$ & $1.091(1.026-1.160)$ & $<0.001$ & $1.091(1.026-1.160)$ & $<0.001$ & \\
\hline
\end{tabular}

Model 1 regression was done with adjustment for age; Model 2 regression was done with adjustment for age, systolic blood pressure, heart rate, estimated glomerular filtration rate, admission time, and hemoglobin. WBC: white blood cell 

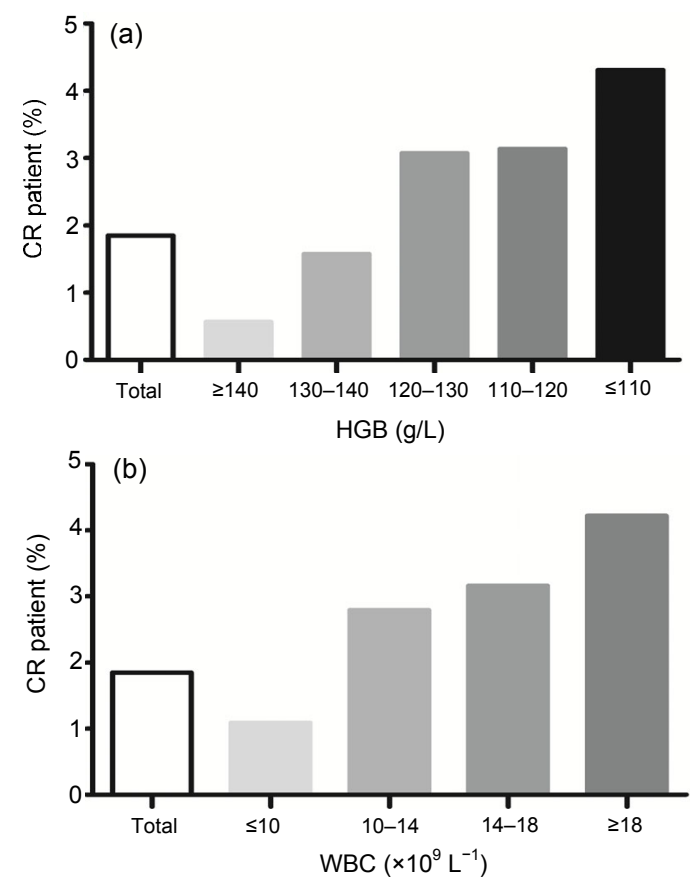

Fig. 1 Relations between cardiac rupture (CR) and anaemia (a) and white blood cell (WBC) levels (b) (a) CR incidence increases with the decline of hemoglobin (HGB): 22 (0.56\%), 35 (1.57\%), 47 (3.07\%), 29 (3.13\%), and $45(4.31 \%)$ for $\geq 140,130-140,120-130,110-120$, and $\leq 110 \mathrm{~g} / \mathrm{L}$ HGB, respectively. (b) CR incidence increases with the increase of WBC levels: $63(1.09 \%), 75(2.79 \%), 28$ $(3.16 \%)$, and $12(4.22 \%)$ for $\leq 10 \times 10^{9}, 10 \times 10^{9}-14 \times 10^{9}$, $14 \times 10^{9}-18 \times 10^{9}$, and $\geq 18 \times 10^{9} \mathrm{~L}^{-1} \mathrm{WBC}$, respectively

\section{Discussion}

CR remains a major cause of mortality after STEMI and persists as a clinic challenge (Gao et al., 2012). The current incidence of rupture reported in the literature is between $1 \%$ and $3 \%$ of patients suffering STEMI (Okino et al., 2005; French et al., 2010; Lopez-Sendon et al., 2010). However, autopsy research detected CR in $30 \%$ to $65 \%$ of patients who died of acute myocardial infarction (AMI) (Hutchins et al., 2002), which indicated that $\mathrm{CR}$ might have been globally underestimated by misdiagnosis. Our study showed the incidence of CR was $1.8 \%$, slightly lower than reported before, partly because of the strict diagnostic criteria in our study. Some sudden unexpected deaths were not diagnosed as CR due to lack of autopsy. The percentage of sudden deaths due to rupture remained high in the first month postinfarction (Pouleur et al., 2010). CR was the second cause of 30-d mortality for STEMI in our study $(16.1 \%, 109 / 676)$, while pump failure was the first cause, which was in accordance with previous reports confirmed by autopsy (Shamshad et al., 2010).

It is necessary to have early and effective risk stratifications in STEMI patients according to risk factors of CR. Our research based on multicenter database was more credible than previous studies, and has investigated some new independent risk factors of $\mathrm{CR}$. We further analyzed some new risk factors such as higher WBC count and lower level of hemoglobin. In particular, hemoglobin level decline independently correlated to occurrence of CR in Chinese STEMI patients after adjustments for other CR risk factors. In CR patients, leukocytes in filtration and hemorrhage are key histopathology (Lewis et al., 1969; Kawano et al., 1994; van den Borne et al., 2009). The intramyocardial dissecting hematoma might appear as a component of the rupture during the remodeling process, and increases the likelihood of CR (Pliam and Sternlieb, 1993; Vargas-Barrón et al., 2013). Both intramyocardial dissecting hematoma and intramyocardial hemorrhage are associated with hemoglobin level decline. On the other hand, pericardial effusion may be present for a few days prior to a fatal rupture (Figueras et al., 2010), implying that CR is a dynamic process. Pericardial effusion was also independently correlated with anemia in our study. In our previous research, we have indicated that risk of CR after AMI was related to the risk of hemorrhage (Qian et al., 2013). Hemorrhage of the myocardium often leads to hemoglobin level decline and bloody pericardial effusion, which could explain why anemia was closely associated with occurrence of CR. Both inflammatory cells in filtration and matrix metalloproteinase-9 (MMP-9) expression were significantly more severe in ruptured than in non-ruptured hearts with acute MI (Fang et al., 2007; van den Borne et al., 2009; Gao et al., 2010), which could explain why the CR group had higher WBC counts in our study.

The prevention of mechanical complications should be emphasized in the acute phase of STEMI. We aimed to establish a risk evaluation model providing a good discrimination in Chinese STEMI patients. An early and effective intervention strategy for $\mathrm{CR}$ prevention is necessary. Early use of $\beta$-blockers and ACEI/ARB could help to prevent CR for patients with high CR risk (Figueras et al., 2008; 
Pedrazzini et al., 2008). Primary PCI therapy rather than thrombolysis could reduce the incidence of $\mathrm{CR}$ (Yip et al., 2003; Okino et al., 2005; Figueras et al., 2008; Qian et al., 2011). The gradual decline in the incidence of CR over a 30-year period was associated with progressive implementation of primary PCI (Figueras et al., 2008). Such knowledge will help facilitate personalized decision-making for patients who are at high risk of CR.

This study has some limitations. CR is an uncommon clinical condition, and thus involves a limited number of cases. This study included a few cases diagnosed based on clinical manifestations rather than necropsy. This was a retrospective cohort study, so retrospective bias exists in the statistics.

\section{Conclusions}

The incidence of CR is low in Chinese STEMI patients, but is associated with poor prognosis. A number of variables are independently related to $C R$. This study pointed out, for the first time, that both hemoglobin and WBC levels were strongly and independently correlated with occurrence of CR.

\section{Acknowledgements}

We sincerely thank Dr. Chang-zhong CHEN (DanaFarber Cancer Institute, Boston, Massachusetts, USA) for providing valuable statistical analysis for this study.

\section{Compliance with ethics guidelines}

Geng QIAN, Chen WU, Yun-dai CHEN, Chen-chen TU, Jin-wen WANG, and Yong-an QIAN declare that they have no conflict of interest.

This article does not contain any studies with human or animal subjects performed by any of the authors.

\section{References}

Fang, L., Gao, X.M., Moore, X.L., et al., 2007. Differences in inflammation, MMP activation and collagen damage account for gender difference in murine cardiac rupture following myocardial infarction. J. Mol. Cell. Cardiol., 43(5):535-544. [doi:10.1016/j.yjmcc.2007.06.011]

Figueras, J., Alcalde, O., Barrabes, J.A., et al., 2008. Changes in hospital mortality rates in 425 patients with acute ST-elevation myocardial infarction and cardiac rupture over a 30-year period. Circulation, 118(25):2783-2789. [doi:10.1161/CIRCULATIONAHA.108.776690]

Figueras, J., Barrabes, J.A., Serra, V., et al., 2010. Hospital outcome of moderate to severe pericardial effusion complicating
ST-elevation acute myocardial infarction. Circulation, 122(19):1902-1909. [doi:10.1161/CIRCULATIONAHA. 109.934968]

French, J.K., Hellkamp, A.S., Armstrong, P.W., et al., 2010. Mechanical complications after percutaneous coronary intervention in ST-elevation myocardial infarction (from APEX-AMI). Am. J. Cardiol., 105(1):59-63. [doi:10. 1016/j.amjcard.2009.08.653]

Gao, X.M., Ming, Z., Su, Y., et al., 2010. Infarct size and post-infarct inflammation determine the risk of cardiac rupture in mice. Int. J. Cardiol., 143(1):20-28. [doi:10. 1016/j.ijcard.2009.01.019]

Gao, X.M., White, D.A., Dart, A.M., et al., 2012. Post-infarct cardiac rupture: recent insights on pathogenesis and therapeutic interventions. Pharmacol. Ther., 134(2):156179. [doi:10.1016/j.pharmthera.2011.12.010]

Hutchins, K.D., Skurnick, J., Lavenhar, M., et al., 2002. Cardiac rupture in acute myocardial infarction: a reassessment. Am. J. Forensic Med. Pathol., 23(1):78-82. [doi:10.1097/00000433-200203000-00017]

Kawano, H., Miyauchi, K., Okada, R., et al., 1994. Histopathological study of cardiac rupture following myocardial infarction with and without thrombolytic therapy. J. Cardiol., 24(4):249-255.

Kushner, F.G., Hand, M., Smith, S.C., et al., 2009. 2009 focused updates: ACC/AHA guidelines for the management of patients with ST-elevation myocardial infarction (updating the 2004 guideline and 2007 focused update) and ACC/AHA/SCAI guidelines on percutaneous coronary intervention (updating the 2005 guideline and 2007 focused update): a report of the American College of Cardiology Foundation/American Heart Association Task Force on practice guidelines. Circulation, 120(22):22712306. [doi:10.1161/CIRCULATIONAHA.109.192663]

Lewis, A.J., Burchell, H.B., Titus, J.L., 1969. Clinical and pathologic features of postinfarction cardiac rupture. Am. J. Cardiol., 23(1):43-53. [doi:10.1016/0002-9149(69) 90240-9]

Lopez-Sendon, J., Gurfinkel, E.P., Lopez de Sa, E., et al., 2010. Factors related to heart rupture in acute coronary syndromes in the global registry of acute coronary events. Eur. Heart J., 31(12):1449-1456. [doi:10.1093/eurheartj/ ehq061]

Markowicz-Pawlus, E., Nozynski, J., Sedkowska, A., et al., 2007. Cardiac rupture risk estimation in patients with acute myocardial infarction treated with percutaneous coronary intervention. Cardiol. J., 14(6):538-543.

Okino, S., Nishiyama, K., Ando, K., et al., 2005. Thrombolysis increases the risk of free wall rupture in patients with acute myocardial infarction undergoing percutaneous coronary intervention. J. Interv. Cardiol., 18(3):167-172. [doi:10.1111/j.1540-8183.2005.04110.x]

Pedrazzini, G., Santoro, E., Latini, R., et al., 2008. Causes of death in patients with acute myocardial infarction treated with angiotensin-converting enzyme inhibitors: findings from the Gruppo Italiano per lo Studio della Sopravvivenza 
nell'Infarto (GISSI)-3 trial. Am. Heart J., 155(2):388-394. [doi:10.1016/j.ahj.2007.10.015]

Pliam, M.B., Sternlieb, J.J., 1993. Intramyocardial dissecting hematoma: an unusual form of subacute cardiac rupture. J. Card. Surg., 8(6):628-637. [doi:10.1111/j.1540-8191. 1993.tb00422.x]

Pouleur, A.C., Barkoudah, E., Uno, H., et al., 2010. Pathogenesis of sudden unexpected death in a clinical trial of patients with myocardial infarction and left ventricular dysfunction, heart failure, or both. Circulation, 122(6):597-602. [doi:10.1161/CIRCULATIONAHA.110. 940619]

Qian, G., Wang, Z.F., Liu, H.B., et al., 2011. Clinical manifestations of 43 patients with acute myocardial infarction complicated by free wall rupture. Chin. J. Cardiovasc. Dis., 39(9):812-815 (in Chinese).

Qian, G., Liu, H.B., Wang, J.W., et al., 2013. Risk of cardiac rupture after acute myocardial infarction is related to a risk of hemorrhage. J. Zhejiang Univ.-Sci. B (Biomed. \& Biotechnol.), 14(8):736-742. [doi:10.1631/jzus.B1200306]
Shamshad, F., Kenchaiah, S., Finn, P.V., et al., 2010. Fatal myocardial rupture after acute myocardial infarction complicated by heart failure, left ventricular dysfunction, or both: the valsartan in acute myocardial infarction trial (VALIANT). Am. Heart J., 160(1):145-151. [doi:10.1016/ j.ahj.2010.02.037]

van den Borne, S.W., Cleutjens, J.P., Hanemaaijer, R., et al., 2009. Increased matrix metalloproteinase -8 and -9 activity in patients with infarct rupture after myocardial infarction. Cardiovasc. Pathol., 18(1):37-43. [doi:10.1016/j.carpath. 2007.12.012]

Vargas-Barrón, J., Roldán, F.J., Romero-Cárdenas, A., et al., 2013. Intramyocardial dissecting hematoma and postinfarction cardiac rupture. Echocardiography, 30(1): 106-113. [doi:10.1111/echo.12017]

Yip, H.K., Wu, C.J., Chang, H.W., et al., 2003. Cardiac rupture complicating acute myocardial infarction in the direct percutaneous coronary intervention reperfusion era. Chest, 124(2):565-571. [doi:10.1378/chest.124.2.565]

\section{中文概要:}

\section{本文题目：急性 ST 段抬高型心肌梗死继发心脏破裂的预测因素分析}

Predictive factors of cardiac rupture in patients with ST-elevation myocardial infarction

研究目的: 心脏破裂是急性 ST 段抬高型心肌梗死最严重的并发症之一, 病情凶险, 死亡率高。通过明 确心肌梗死后出现心脏破裂并发症的危险因素, 早期识别心脏破裂高危人群, 有利于在急 性心肌梗死的早期采取干预措施以减少心脏破裂的发生。

创新要点: 既往缺乏对中国 ST 段抬高型心肌梗死继发心脏破裂的系统风险评估, 本研究采用回顾性队 列研究的方法, 完善并健全中国多中心的急性心肌梗死数据库, 样本总量达到 9798 例, 为 国内同类研究中样本量最多。既往的国外文献报道: 女性、高龄、心梗后就诊时间延迟、 前壁心肌梗死、溶栓治疗等是心肌梗死后心脏破裂的独立危险因素。本研究在中国人群验 证以上危险因素的基础上首次提出就诊时血色素下降及白细胞计数的升高与随后发生的心 脏破裂密切相关。新的心脏破裂预测因素的发现有利于对心脏破裂更精细化的危险分层, 早期识别心脏破裂高危人群。

研究方法: 建立中国多中心心肌梗死数据库, 比较发生心脏破裂与未发生心脏破裂患者临床特点的差 异，篮选敏感特异的心脏破裂的独立预测因素。

重要结论: 中国人群急性 ST 段抬高型心肌梗死后心脏破裂的发生率是 $1.82 \%$, 独立预测因素主要包括: 高龄、女性、就诊时静息心率增快、前壁心肌梗死、心功能不全、就诊时间延迟、肾功能 不全、贫血与白细胞计数升高。本文第一次提出就诊时贫血状态及白细胞计数的升高时与 心脏破裂的发生直接相关。

关键词组：ST 段抬高型心肌梗死；危险因素；贫血；心脏破裂 